\title{
MORPHOLOGY AND MECHANICAL PROPERTIES OF THE SUBRENAL AORTA IN NORMOTENSIVE AND HYPERTENSIVE RATS
}

\author{
Petra Kochova $^{a *}$, Zbynek Tonar ${ }^{\mathrm{a}, \mathrm{b}, \mathrm{c}}$, Vit M. Matejka ${ }^{\mathrm{b}}$, Jitka Sviglerova ${ }^{\mathrm{d}}$, Milan Stengl, \\ Jitka Kuncova ${ }^{\mathrm{d}}$
}

a Department of Mechanics, Faculty of Applied Sciences, University of West Bohemia, Univerzitni 22, 30614 Pilsen, Czech Republic

${ }^{b}$ Department of Histology and Embryology Faculty of Medicine in Pilsen, Charles University in Prague, Karlovarska 48, 30166 Pilsen, Czech Republic

c Laboratory for Atherosclerosis Research, Institute for Clinical and Experimental Medicine, Videnska 1958/9, 14021 Prague 4, Czech Republic

d Department of Physiology, Faculty of Medicine in Pilsen, Charles University in Prague, Plzen, Lidicka 1, 30166 Pilsen, Czech Republic

e-mail: kochovap@kme.zcu.cz

Received: October 16, 2008; Accepted (with revision): December 5, 2008

Key words: Rat subrenal aorta/Arterial wall/Hypertension/Remodelling/Mechanical measurement/Mechanical properties

Aims: This work focuses on the morphological and mechanical changes in the wall of the subrenal aorta in rats suffering from arterial hypertension and chronic renal failure induced by subtotal nephrectomy (NX).

Methods: The quantitative structural parameters were assessed using stereological methods. The mechanical properties were determined using uniaxial tensile tests.

Results: Morphological results showed no significant differences in the wall structure of NX rats ten days following the subtotal nephrectomy in comparison with the control animals. Pronounced structural changes appeared ten weeks after the nephrectomy. The area of the profile of the arterial lumen, the volume fraction of elastin, and the elastin lamellar number in the tunica media were significantly higher in the NX rats than in the control animals. The values of the volume fraction of the smooth muscle cells in the tunica media and the lamellar unit thickness were significantly lower for the NX animals. Mechanical results showed that both kinds of tissues were characterized by a non-linear response when subjected to the tensile test. The moduli of elasticity of subrenal aortas in control and NX animals were different only for large deformations: NX samples had higher stiffness. The cyclic loading resulted in a time-dependent response for both tissue types.

Conclusions: The results obtained from the NX rats ten days as well as ten weeks after operation suggested an outward hypertrophy of the aorta. The subrenal aortas of the NX animals had less strength and were less extensible than those of the control animals.

\section{INTRODUCTION}

\section{Mechanical Properties of Soft Biological Tissues}

The hierarchical structure of biological tissues has a significant influence on their mechanical behaviour. Minns et al. compared the stress-strain relationships of the human Achilles tendon, human ascending aorta, and bovine ligamentum nuchae ${ }^{9}$. They found that tissues with the highest percentage of collagen have the highest strength, but the ultimate (failure) strengths were not directly proportional to the collagen content (tendon $86 \%$, aorta $12-24 \%$ and ligament $16.3 \%$ of the dry weight), possibly due to differences between the fibre arrangements and inter-fibre bonding.

The mechanical properties of materials can be determined from their behaviour under mechanical loading, such as stress or strain loading. Stress is defined as a force divided by the cross-sectional area. Strain is defined as the difference between the actual and initial dimension (length) divided by the initial dimension. Biological tissues under normal physiological conditions subjected to cyclic mechanical loading exhibit large displacements, as a result of their physiological nature. The mechanical aspects of living tissues, including the veins and arteries allow them to be classified as hyperelastic materials.

The majority of soft biological materials, including arteries, do not have simple linear elastic properties, meaning that the relation between stress and strain is not a straight line. The elastic stiffness increases with the degree of loading ${ }^{14,16}$. There are three major regions of the stress-strain curve: the toe region, the heel region, and the linear region. It is also possible to detect the yield and failure regions. The toe region, where few collagen fibres are straight, represents the "un-crimping" of the crimp in the collagen fibres. In this region, the components responsible for the elastic behaviour are the elastin fibres. Since it is easier to stretch out the crimp of the collagen fibres, this part of the stress-strain curve shows a relatively low 
stiffness. In the heel region, the majority of the fibres are straight. As the collagen fibres become un-crimped, the collagen fibre backbone is stretched, which gives causes the material to stiffen. The linear region is characterised by all of the collagen fibres being straight. As the individual fibres within the arteries fail and damage accumulates, the stiffness is reduced and the arteries begin to fail.

Other important mechanical aspects of arterial behaviour are viscoelasticity and hysteresis ${ }^{5}$. Viscoelasticity indicates that the relationship between stress and strain depends on the time history of the displacements or loading. Hysteresis, also known as energy dissipation, means that if a viscoelastic material is loaded and unloaded, the unloading curve will not follow the loading curve. This difference between the two curves represents the amount of energy that is dissipated or lost during loading. Under cyclic loading, the hysteresis is progressively reduced and eventually the stress-strain curve becomes reproducible. In other words, by increasing the number of cycles, smaller stresses are needed to stretch the aorta to the same elongation. If the test is repeated indefinitely, the difference between successive cycles decreases, and eventually disappears. Specimens subject to this phenomenon are said to have been preconditioned. The reason that preconditioning occurs in a specimen is that the internal structure of the tissue changes during the cycling.

\section{Hypertension and Remodelling of Arterial Tissue}

Hypertension is a common disease that leads to remodelling of the arterial tissue, which increases the diameter and the intima-media thickness in both elastic and muscular arteries ${ }^{4,8,15}$. High arterial blood pressures lead to changes in the area of the profile of arterial wall, area of the profile of arterial lumen, and the volume fractions of the constituents in the arterial wall. Remodelling can be hypertrophic with an increased cross-sectional area, eutrophic with no change in cross-sectional area, or hypotrophic with a decreased cross-sectional area ${ }^{11}$. These three groups can be further classified in terms of inward and outward remodelling. The remodelling involves the detection of mechanical or chemical signals from the cells, relaying these signals within the cells, synthesis, activation or release of substances that influence cellular or noncellular processes, and finally structural and functional changes in the vessel $1^{17,22}$.

The onset and time-related nature of these changes have not been described in hypertensive rats. To elucidate these changes, morphological changes in the wall structure of rats after ten days and ten weeks were observed following induction of hypertension and chronic renal failure (CRF) by subtotal nephrectomy. The results were compared with those obtained in control animals. Moreover, there is a lack of information explaining how these changes influence the mechanical properties of the vessels. In order to clarify this dependency, this work investigates the changes in the mechanical properties in the wall of the subrenal aorta of NX rats ten weeks after hypertension was induced. The behaviour of NX and control tissues under uniaxial mechanical loading was studied,
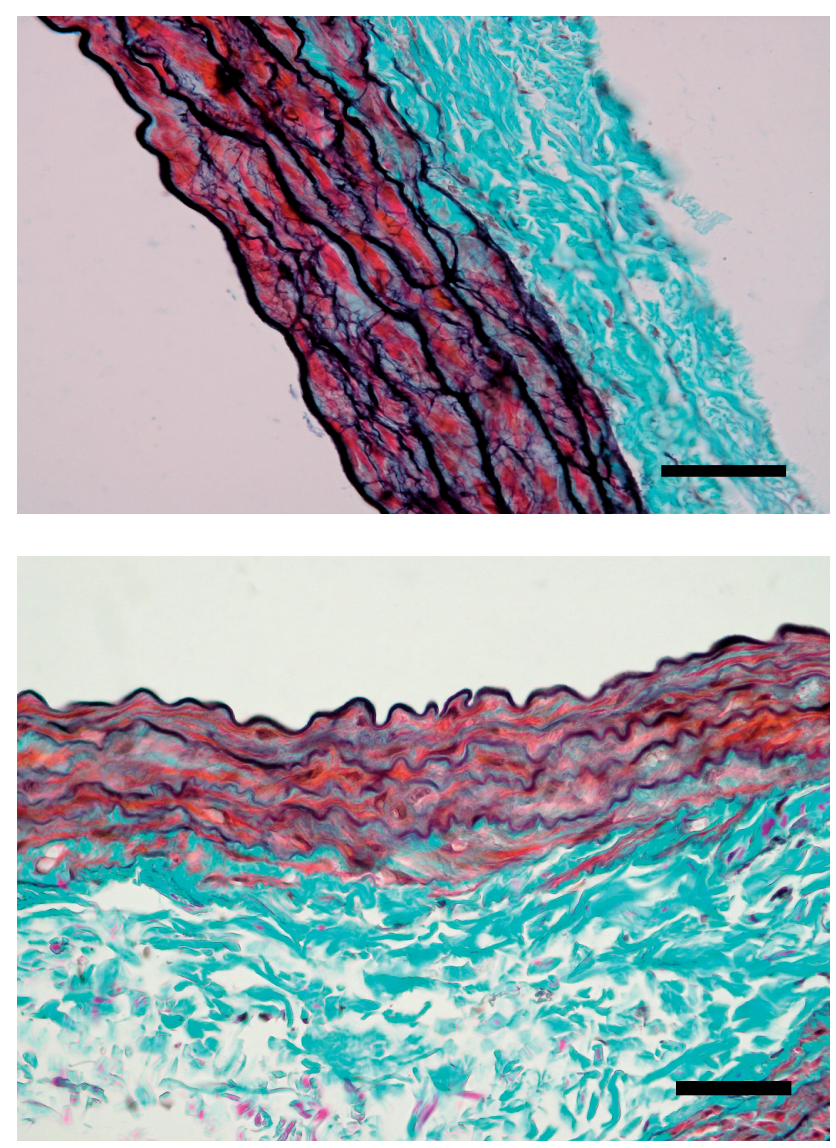

Fig. 1. (a,b) Wall of subrenal aorta in rats with subtotal nephrectomy (top) and following sham operation (bottom). Trichrome staining. Scale bars represent $50 \mu \mathrm{m}$.

and the mechanical parameters were determined. The values for the control and NX animals were compared.

\section{MATERIAL AND METHODS}

Changes to the morphological properties of subrenal aorta of subtotally nephrectomized rats 10 days as well as 10 weeks following the induction of CRF or sham (control) operation were investigated. Moreover, the mechanical properties of the subrenal aorta of NX and sham operated rats 10 weeks following operation were determined for comparison.

\section{Animal Preparation and Surgery}

All animal experiments were conducted in accordance with the European Directive for the Protection of Vertebrate Animals Used for Experimental and Other Scientific Purposes (86/609/EU), the relevant Guidelines of the Czech Ministry of Agriculture for scientific experimentation in animals, and were approved by the University Committee for Experiments in Laboratory Animal. Sixteen male 4-month-old Wistar rats were randomly allocated to the subtotal (5/6) nephrectomy (NX) and sham operation (control) groups. CRF was induced by 
the complete removal of the upper and lower poles of the right kidney, followed by the complete removal of the left kidney after two weeks. The control animals were sham operated by exteriorizing the kidney and subsequently replacing the intact kidney back into the abdominal cavities. The rats were anesthetized with pentobarbital $(100 \mathrm{mg} / \mathrm{kg}$ intraperitoneally) during the surgical procedures.

\section{Histology}

Either ten days or ten weeks following subtotal nephrectomy or sham operation, the rats were anesthetized with urethan ( $1.5 \mathrm{~g} / \mathrm{kg}$ body weight) and killed by decapitation. The entire descending aorta was dissected, weighed and separated below the left renal artery into two parts. A $2 \mathrm{~mm}$ segment of the subrenal aorta was removed for histological processing, perfused with formalin under $60 \mathrm{~mm} \mathrm{Hg}$ for $30 \mathrm{~min}$ and embedded in paraffin. Each sample was cut into 72 serial sections with a thickness of $5 \mu \mathrm{m}$. We used green trichrome and Verhoeff's hematoxylin for staining, as illustrated in Fig. 1.

\section{Quantitative Microscopy}

In order to describe the morphological changes of the aortic wall, seven quantitative parameters were used: cross-sectional area of the arterial lumen, intima media thickness, volume fraction of smooth muscle cells (SMC) in the tunica media, volume fraction of elastin in the tunica media, lamellar number of elastin fibres, lamellar unit thickness of elastin fibres, and length density of profiles of elastin lamellae. Stereological methods were used for these quantifications ${ }^{10,13}$. The Ellipse software (ViDiTo, Inc., Košice, SR) was used for the stereological measurement. Within the reference volume, equidistant sections were selected through systematic uniform random sampling. The position of the first tissue section in the series was random, i.e., equal to a product of $(72 * n)$, where $n$ was a random number between 0 and 1 . Every sixth section was captured and analysed.

We assessed the area of the profile of the arterial lumen and the area of profiles of elastin and SMC in the tunica media in each tissue section according to

$$
\text { est } A=a \cdot P \text {, }
$$

where $e s t A$ was the estimated area of the profile of the lumen, elastin, or SMC; the grid parameter $a$ was the area corresponding to one test point; and $P$ was the number of test points hitting the relevant structure. The total number of points counted was at least 200 in all sections of each series. The Cavalieri principle was used to estimate the volume of the tissue within the reference segment of the $\operatorname{aorta}^{7,10}$ :

$$
\text { est } V=T \cdot \sum_{i=1}^{m} A_{i} \text {, }
$$

where $e s t V$ was the Cavalieri volume estimator; $T=0.045$ $\mathrm{mm}$ was the distance between the two subsequent selected sections; $A_{i}$ was the area estimated using (1) in the $i$-th section; $m$ represented the total number of sections se-

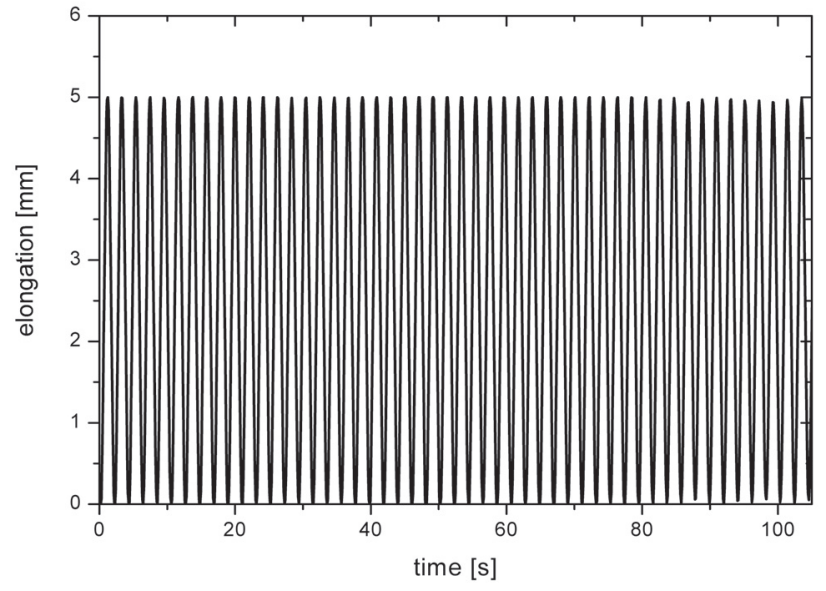

Fig. 2. The uniaxial cyclic loading (50 cycles) of a subrenal aorta. One cycle consisted of a linearly growing elongation up to $5 \mathrm{~mm}$ and a symmetrical shortening back to the initial length of the specimen. The loading velocities were 400 and $-400 \mathrm{~mm} / \mathrm{min}$ for the first and the second parts of the cycle, respectively.

lected from the series. To estimate the value of $m$, that was, the variation caused by sampling the serial sections, Gundersen and Jensen's method was used to predict the coefficient of error (CE) (ref. $^{6}$ ). Preliminary analysis proved that the number of sections sampled within each tissue block had to be $m=12$ in order to maintain the $\mathrm{CE}=0.05$.

The volume fraction of elastin within the tunica media $V_{V}$ (elastin,media) and volume fraction of SMC within the tunica media $V_{V}(S M C$, media $)$ were calculated using the relation

$$
V_{V}(Y, \text { media })=\frac{V_{Y}}{V},
$$

where $V_{Y}$ was the volume of elastin or SMC in reference space (the tunica media) and $V$ was the volume of reference space.

The intima-media thickness (IMT) was assessed as a mean value of the length of four testing lines applied in a systematic uniform random way on the profile of the intima and the media, appearing on transversal sections through each artery.

The lamellar number (LN) represented the number of elastin lamellae of the tunica media counted in the direction of radial axis. The minimum thickness of elastin profiles that could be counted as elastin lamella (equal to or greater than $1.83 \mu \mathrm{m}$ ) was defined for this measurement, to avoid counting the smallest branches of the main elastin lamellae.

The lamellar unit thickness (LUT) representing the mean interlamellar distance between adjacent profiles of elastin lamellae was defined as:

$$
L U T=\frac{I M T}{L N}
$$


Table 1.

\begin{tabular}{|l|c|c|c|c|}
\hline parameter & SHAM 10D & NX 10D & SHAM 10W & NX 10W \\
\hline$V_{V}$ (elastin,media) & $0.44 \pm 0.07$ & $0.45 \pm 0.05$ & $0.37 \pm 0.11$ & $0.40 \pm 0.12^{*}$ \\
\hline$V_{V}($ SMC,media $)$ & $0.50 \pm 0.06$ & $0.51 \pm 0.04$ & $0.55 \pm 0.12$ & $0.50 \pm 0.12^{*}$ \\
\hline IMT $(\mu \mathrm{m})$ & $109.71 \pm 14.43$ & $109.18 \pm 6.60$ & $106.95 \pm 11.49$ & $105.78 \pm 11.03$ \\
\hline LN & $5.24 \pm 1.13$ & $5.43 \pm 0.55$ & $5.60 \pm 1.45$ & $6.33 \pm 1.51^{*}$ \\
\hline LUT $(\mu \mathrm{m})$ & $22.27 \pm 7.61$ & $20.33 \pm 2.44$ & $21.00 \pm 8.69$ & $18.48 \pm 9.00^{*}$ \\
\hline$L_{A}($ elastin,media $)\left(\mu \mathrm{m}^{-1}\right)$ & $0.14 \pm 0.01$ & $0.15 \pm 0.01^{*}$ & $0.15 \pm 0.04$ & $0.15 \pm 0.03$ \\
\hline
\end{tabular}

Morphometric parameters of the subrenal aorta wall of rats subjected to 5/6 nephrectomy (NX) or sham operation (SHAM) in 10 days (10D) and ten weeks (10W) following the operation. No significant differences in values of the volume fraction of elastin in the tunica media $\left(V_{V}\right.$ (elastin, media)), the volume fraction of smooth muscle cells in the tunica media ( $V_{V}(S M C$, media $)$ ), the thickness of intima-media (IMT), the lamellar unit thickness (LUT) of elastin lamellae and lamellar number (LN) of elastin lamellae were found in ten days following the operation. In ten days following the operation, the length density of profiles of elastin lamellae in the media ( $L_{A}$ (elastin, media)) was slightly higher for the NX rats in comparison to control animals. The volume fraction of elastin in the tunica media and the lamellar number of elastin lamellae were significantly higher in the NX rats compared to the sham operated rats in ten weeks following operation. The volume fraction of smooth muscle cells in the tunica media and the lamellar unit thickness of elastin lamellae were significantly lower in the NX rats in ten weeks following operation. Ten weeks after operation, no differences in the length density of profiles of elastin lamellae in the media and in the thickness of intima-media were found. Data are means \pm standard deviation, ${ }^{*} \mathrm{p}<0.05$.

The 2-D length density of profiles of the elastin lamellae $L_{A}($ elastin,media) was defined as the ratio of the estimated length of elastin profiles (est $L$ ) and the reference area of the tunica media $(A)$. Our method involved counting of the intersections of the elastin fibres and membranes using a network of circular arcs that was randomly superposed on the micrographs.

\section{Mechanical Measurement}

Ten weeks following the subtotal nephrectomy or sham operation, the rats were anesthetized by urethan $(1.5 \mathrm{~g} / \mathrm{kg}$ body weight) and killed by decapitation. Five subrenal aortas from the sham operated (control) animals and three subrenal aortas of NX animals were exposed to uniaxial cyclic mechanical loading. A traction machine (Zwick/Roell GmbH \& Co, Ulm, Germany) equipped with pneumatic grips and a $200 \mathrm{~N}$ loading head was used for the mechanical testing. This device enables the measurement of large deformations in specimens finished by a tissue rupture. The elongation of the sample is prescribed and the forces needed to reach this elongation are measured. The specimens were fixed between the grips of the apparatus with a free length corresponding to their dimensions (13-26 and 19-22 mm for the control and NX subrenal aortas, respectively).

The specimens were subjected to 50 cycles of loading in the longitudinal direction to the main aortic axis, as illustrated in Fig. 2. One cycle consisted of a linearly growing elongation up to $5 \mathrm{~mm}$ and a symmetrical shortening back to the initial length of the specimen. The loading velocities were 400 and $-400 \mathrm{~mm} / \mathrm{min}$ for the first and the second parts of the cycle, respectively. This elongation rate is the highest velocity that can be produced by the testing apparatus. After this preconditioning, the tensile test with a linearly growing elongation was applied until the tissue ruptured. The stress-strain curves were recorded. The moduli of elasticity for each cycle (cyclic loading), the moduli of elasticity for tension, ultimate strength, and ultimate strain (tensile test) were all determined. The programming language Python which includes libraries for the least squares method regression was used to evaluate the stress-strain curves ${ }^{12}$.

\section{Statistical Analysis}

Statistica Base 7.1 (StatSoft, Inc., Tulsa, OK, USA) was used for data processing. The normality of the data was tested with the Shapiro-Wilk W test. The Levene and Brown-Forsythe tests were used to assess variance homogeneity. The differences between the NX and control groups were evaluated using Student's unpaired t-test. The results were considered significantly different when $p<0.05$.

\section{RESULTS}

\section{Quantitative Analysis}

Ten days after the operation, no significant differences were detected between the NX and sham operated group with respect to the intima-media thickness $(p=0.872)$, volume fraction of SMC $V_{v}(S M C$, media $)(p=0.615)$, volume fraction of elastin $V_{v}$ (elastin, media) $(p=0.802)$, lamellar unit thickness $(p=0.241)$, and lamellar number $(p=0.463)$. However, the value of length density of the 
elastin profiles was higher for the NX rats than for control animals $(p=0.006)$. The actual values of all investigated morphological parameters 10 days following the operation are presented in Table 1. The cross-sectional area of the arterial lumen 10 days after operation was $7.3 \pm 2.7$ (means \pm standard deviation) in the control and $8.9 \pm$ $1.8 \mathrm{~mm}^{2}$ in the NX animals, respectively.

Ten weeks following the operation, a significantly lower SMC volume fraction $V_{v}(S M C$, media) was detected in the NX rats compared to the control animals $(p<0.001)$. By contrast, NX animals exhibited a significantly higher value for the elastin volume fraction $V_{v}$ (elastin, media) than did the control animals $(p<0.001)$. No significant differences were found in the values of length density of elastin profiles and intima-media thickness between the two groups of rats $(p=0.126$ and $p=0.212$ for the NX and control specimens, respectively). However, the lamellar unit thickness was significantly lower $(p<0.001)$ and lamellar number significantly higher $(p<0.001)$ in the NX than in the sham operated rats. The actual values of all investigated morphological parameters 10 weeks following operation are presented in Table 1 . The cross-sectional area of the arterial lumen 10 weeks after operation was $7.3 \pm 2.3$ in the control and $11.8 \pm 3.8 \mathrm{~mm}^{2}$ in the NX animals, respectively.

\section{Cyclic Loading}

The stress-strain curves for the cyclic loading are given in Fig. 3. The time-dependence (hysteresis) of the tissue response on the cyclic loading was visible with increasing number of cycles for both kinds of tissue. The results show that a lower force was necessary to reach the same specimen extension with an increasing number of cycles. The maximal stresses of the hysteresis curves decreased and the tissue tended towards a steady state.

The first part of each cycle was approximated using the linear regression to obtain the moduli of elasticity. The dependencies of the moduli of elasticity on the number of cycles are shown in Figs. 4 and 5 for the subrenal aorta of the control and NX animals, respectively. It is evident that the values for the moduli of elasticity decreased with increasing number of cycles in both cases.

The subrenal aortas of the NX animals had an initial value above $0.2 \mathrm{MPa}$ and as the number of cycles increased, approached a value of about $0.12 \mathrm{MPa}$. Conversely, the control samples of the subrenal aortas initially exhibited a higher value of the modulus of elasticity above $0.22 \mathrm{MPa}$. This value then decreased relatively quicker than that for the NX samples and after about 10 cycles approached the stable value of about $0.12 \mathrm{MPa}$, similar to that obtained for the NX samples. In other words, the differences between the control and NX samples were visible in the first cycles but then disappeared.

\section{Tensile Test}

The linearly growing elongation of the specimen resulted in a non-linear stress-strain curve. The toe, heel, and linear regions were significantly visible, as shown in Figs. 6 and 7 . The toe and linear regions were approximated using

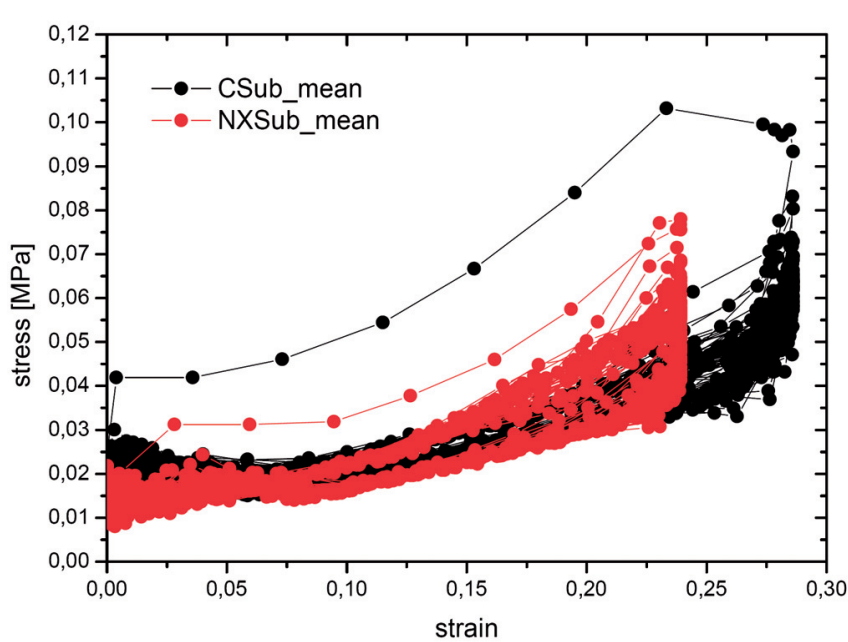

Fig. 3. The response of the subrenal aortas of control sham operated (black) and NX (red) animals. The hysteresis when the sample was loaded and unloaded is visible. The energy loss given by the area between the loading and unloading curves for each cycle decreased as the number of cycles increased and approached a constant value, meaning the sample was preconditioned.

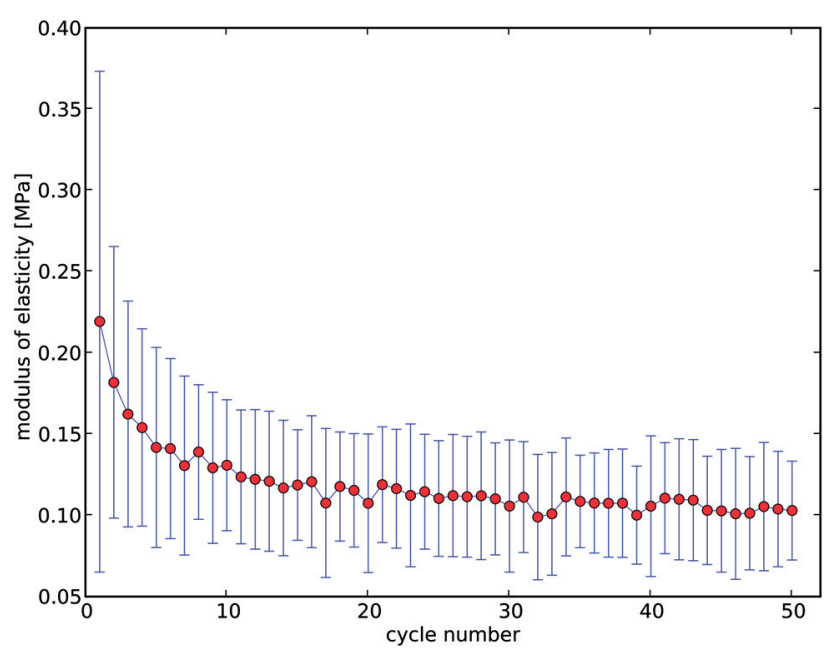

Fig. 4. The resultant values of moduli of elasticity of the subrenal aortas of control sham operated animals. The decrease of modulus of elasticity with an increasing number of cycles is visible. The mean value \pm standard deviation $(n=5)$.

a linear regression and the moduli of elasticity were determined. The values of the moduli of elasticity were about 0.09 and $2.86 \mathrm{MPa}$, for the toe and linear regions of subrenal aortas in sham operated animals, respectively (Fig. 6). For the NX subrenal aortas, the moduli of elasticity were $0.09 \mathrm{MPa}$ for the toe region and 3.10 MPa for the linear region (Fig. 7). The values of the ultimate strains were about $85 \%$ and $68 \%$ for subrenal aortas of the sham operated and NX animals, respectively. The ultimate strengths before the tissue rupture were 0.93 and $1.13 \mathrm{MPa}$ for the NX and sham operated animals, respectively. 


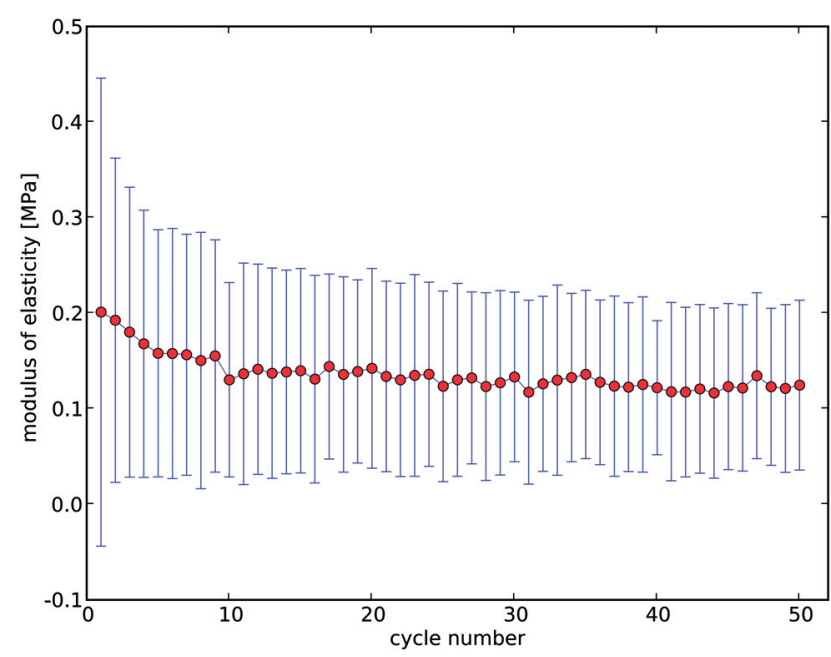

Fig. 5. The resultant values of moduli of elasticity of the subrenal aortas in NX rats 10 weeks following the operation. The decrease of modulus of elasticity with an increasing number of cycles is visible. The mean value \pm standard deviation $(n=3)$.

\section{DISCUSSION}

The histomorphometric analysis was performed on the wall of the subrenal aorta in the animals that acquired hypertension induced by $5 / 6$ nephrectomy. The results obtained from the NX rats 10 days as well as 10 weeks following the operation suggested an outward hypertrophy of the aorta of subtotally nephrectomized rats, as evidenced by the increased weight of the vessel that was not accompanied by an increased vessel wall thickness. The analysis demonstrated that the wall structure of the subrenal aorta is rearranged over time in rats with induced hypertension. The changes of the structural parameters were not significant 10 days following operation, except for an increased length density of elastin profiles in NX rats. The volume fraction of SMC as well as elastin, the lamellar number of elastin and lamellar thickness of elastin changed rapidly during the 10 weeks following the operation.

The mechanical experiments demonstrated that the loading and unloading occur on different stress-strain paths. This hysteresis in the stress-strain relationship clearly showed the viscoelastic behaviour of the subrenal aorta. The stress-strain curves were visibly shifted with an increasing number of cycles for both types of subrenal walls. The energy dissipated in the first cycle was significantly higher for the control samples in comparison to the NX samples.

The values of moduli of elasticity of subrenal aorta of the NX samples were only different than the control samples for a few starting cycles. The values for the NX samples could be influenced by the high variance between the individual NX samples, as shown in Fig. 7.

The subrenal aortas of rats loaded in the longitudinal direction to the main aortic axis exhibited non-linear behaviour when subjected to a tensile loading in the both cases (subrenal aortas of control and NX animals). The values obtained showed that the subrenal aortas ex-

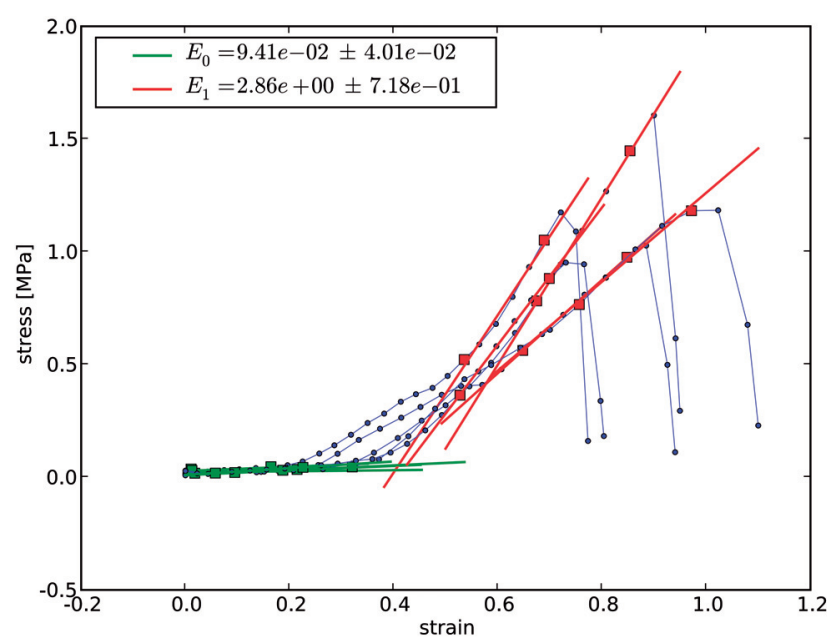

Fig. 6. The stress-strain characteristics of the subrenal aorta of control sham operated animals with visible toe, heel, and linear regions. The toe and linear regions were approximated using a linear regression and the values of moduli of elasticity were determined. $E_{0}$ represents the modulus of elasticity for the toe region and $E_{l}$ represents the modulus of elasticity for the linear region.

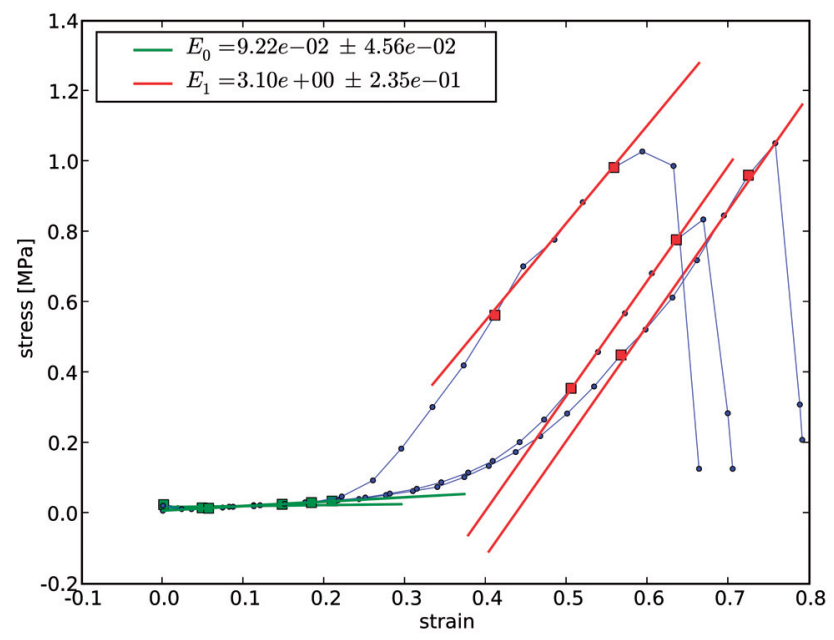

Fig. 7. The stress-strain characteristic of subrenal aorta of NX rats with visible toe, heel, and linear regions. The toe and linear regions were approximated using a linear regression and the values of moduli of elasticity were determined. $E_{0}$ represents the modulus of elasticity for the toe region and $E_{1}$ represents the modulus of elasticity for the linear region.

hibited a loading stiffening characterized an increased Young's modulus for large deformations with respect to the Young's modulus for smaller deformations, similar to the thoracic aorta of pigs described in Angouras et al. ${ }^{2}$. This progressive stiffening due to the increasing load is typical for soft tissues such as tendon, high fascia, skin, ligament, arteries, and aorta ${ }^{3,9,18-21}$. It is possible to detect 
the toe, heel, and linear regions of the stress-strain curve with the significant stiffening of the tissue. These results are in agreement with the fact that the stiffening of the tissue is caused by straightening of the elastin and collagen fibres.

The values of moduli of elasticity of the subrenal aortas of the NX compared to the control samples were not significantly different in the region of small deformations. On the other hand, the moduli of elasticity of the NX samples were significantly higher in the region of large deformations than in the aortas in control sham operated animals. It is necessary to note that the values were probably influenced by the fact that it was impossible to measure the real cross-section of each sample undergoing the mechanical test and therefore the mean values of the aortas cross-sections obtained from stereological measurements were used to evaluate the stress values. Namely, the cross-sections were 5.53 and $4.44 \mathrm{~mm}^{2}$ for all the control and NX subrenal aortas, respectively.

The extensibility for both the kinds of subrenal aortas, characterized by the ultimate strain ( $85 \%$ and $68 \%$ for NX and sham operated, respectively) was higher than that for human tendons (10-15\%). This was comparable to values characteristic for samples of other organs composed of smooth muscle and connective tissue, such as the ureter $(60 \%)$, arteries $(60 \%)$, veins $(60 \%)$, and skin (60-110\%) (ref., ${ }^{18}$ ).

The ultimate strengths were significantly higher for control animals (1.13 MPa) in comparison to the NX animals $(0.93 \mathrm{MPa})$. In both cases the values were smaller than in human tendons (50-100 $\mathrm{MPa})$ and skin (4 MPa), higher than in skeletal muscles $(0.3 \mathrm{MPa})$, and comparable to the ultimate strength of abdominal aorta (1.2 $\mathrm{MPa})$ (ref. ${ }^{5,18}$ ).

\section{CONCLUSION}

The changes in mechanical properties of subrenal aortas of subtotally nephrectomized rats 10 weeks following the induction of chronic renal failure or sham operation were investigated. The resulting mechanical responses in connection with the differences in the composition and the tissue architecture elucidated the magnitude of the effect of structural changes on the mechanical properties of the tissue.

The values of ultimate strength and ultimate strain showed that the subrenal aortas of the NX animals had less strength and were less extensible. Further, the results showed that, under mechanical uniaxial loading with linearly growing elongation, the subrenal aortas of the NX animals in comparison to the control animals had a comparable modulus of elasticity for small deformations and a higher modulus of elasticity for large deformations.

\section{ACKNOWLEDGEMENT}

This work was supported by the Ministry of Education, Youth and Sports of the Czech Republic under the Projects No. MŠM4977751303, MŠM0021620819, and 1M6798582302.

\section{REFERENCES}

1. Ahlfors JEW, Billiar KL. Biomechanical and biochemical characteristics of a human fibroblast-produced and remodeled matrix. Biomaterials 2007; 28:2183-2191.

2. Angouras D, Sokolis DP, Dosios T, Kostomitsopoulos N, Boudoulas $H$, Skalkeas $G$, et al. Effect of impaired vasa vasorum flow on the structure and mechanics of thoracic aorta: Implications for the pathogenesis of aortic dissection. Eur J Cardiothorac Surg 2000; 17:468-476.

3. Belkoff SM, Haut RC. A structural model used to evaluate the changing microstructure of maturing rat skin. J Biomech 1991; 24(8):711-720.

4. Durmowicz AG, Stenmark KR. Mechanism of structural remodeling in chronic pulmonary hypertension. Pediatr Rev 1999; 20:e91e102.

5. Fung YC. Biomechanics. Mechanical Properties of Living Tissues $2^{\text {nd }}$ ed. Springer-Verlag: New York; 1993.

6. Gundersen HJG, Jensen EB. The efficiency of systematic sampling in stereology and its prediction. J Microsc 1987; 147:229-263.

7. Howard CV, Reed MG. Unbiased Stereology: Three Dimensional Measurement in Microscopy. Royal Microscopical Society: New York; 1998.

8. Intengan HD, Schiffrin EL. Vascular remodeling in hypertension. Roles of apoptosis, inflammation, and fibrosis. Hypertension 2001; 38:581-587.

9. Minns RJ, Soden PD. The role of fibrous components and ground substance in the mechanical properties of biological tissues: A preliminary investigation. J Biomech 1973; 6:153-165.

10. Mouton PR. Principles and Practices of Unbiased Stereology: An Introduction for Bioscientists. The Johns Hopkins University Press:Baltimore; 2001.

11. Mulvany MJ. Vascular remodelling of resistance vessels: can we define this? Cardiovasc Res 1999; 41:9-13.

12. Python home page. http://www.python.org, 2008.

13. Russ JC, Dehoff RT. Practical Stereology. $2^{\text {nd }}$ ed. Plenum Press: NewYork; 1999.

14. Safar ME, Blacher J, Moudar JJ, London GM. Stiffness of carotid artery wall material and blood pressure in humans. Stroke 2000; 31:783-790.

15. Sasamura H, Shimizu-Hirota R, Saruta T. Extracellular matrix remodeling in hypertension. Curr Hypertens Rev 2005; 1:51-60.

16. Shadwick RE. Mechanical design in arteries. J Exp Biol 1999; 202:3305-3313.

17. Stenmark KR, Orton EC, Reeves JT, Voelkel NF, Crouch EC, Parks $\mathrm{WC}$, et al. Vascular remodeling in neonatal pulmonary hypertension. Chest 1988; 93:127S-133S.

18. Viidik A. Biomechanical behavior of soft connective tissues. In: Akkas N, editor. Progress in Biomechanics. Hardbound Sijthoff \& Nordhoff; 1979. p. 75-113.

19. Vito RP, Hickey J. The mechanical properties of soft tissues - II: The elastic response of arterial segments. J Biomech 1980; 13(11):951597.

20. Woo SLY, Gomez MA, Woo YK, Akeson WH. Mechanical properties of tendons and ligaments. Biorheology 1982; 19:397-408.

21. Yuan K, Kononov S, Cavalcante FSA, Lutchen KR, Ingenito EP, Suki B. Effect of collagenase and elastase on the mechanical properties of lung tissue strips. J Appl Physiol 2000; 89:3-14.

22. Zervoudaki AI, Toutouzas PK. Remodeling of resistance vessels in essential hypertension. HJC 2003; 44:116-124. 
